\title{
A new approach with new solutions to the Matkowski and Wesołowski problem
}

\author{
Janusz MorawieC(i) AND Thomas Zürcher
}

\begin{abstract}
Based on a result of de Rham, we give a family of functions solving the Matkowski and Wesołowski problem. This family consists of Hölder continuous functions, and it coincides with the whole family of solutions to the Matkowski and Wesolowski problem found earlier by a different method. Moreover, applying some results due to Hata and Yamaguti and due to Berg and Krüppel, we prove that there are functions solving the Matkowski and Wesołowski problem that are not Hölder continuous.
\end{abstract}

Mathematics Subject Classification. Primary 39B12; Secondary 26A16, 26A30.

Keywords. Functional equations, Hölder continuity, singular functions.

\section{Introduction}

In 1985 Janusz Matkowski posed the problem asking if the equation

$$
\varphi(x)=\varphi\left(\frac{x}{2}\right)+\varphi\left(\frac{x+1}{2}\right)-\varphi\left(\frac{1}{2}\right)
$$

has a non-linear monotonic and continuous solution $\varphi:[0,1] \rightarrow \mathbb{R}$ (see [14]). During the 47th International Symposium on Functional Equations in 2009 Jacek Wesołowski asked whether the identity on $[0,1]$ is the only increasing and continuous solution $\varphi:[0,1] \rightarrow[0,1]$ of $(\mathrm{e})$ satisfying

$$
\varphi(0)=0 \quad \text { and } \quad \varphi(1)=1 .
$$

This question of Jacek Wesołowski was posed in connection with studying probability measures in the plane that are invariant under "winding" (see $[15])$.

It is not difficult to check that Matkowski's problem is equivalent to Wesołowski's question (see [16]). A negative answer to Wesołowski's question 
(as well as a positive answer to Matkowski's problem) was obtained in [10] and reads as follows.

Theorem 1.1. (i) The identity on $[0,1]$ is the only increasing and absolutely continuous solution $\varphi:[0,1] \rightarrow[0,1]$ of (e) satisfying (1).

(ii) For every $p \in(0,1)$ the function $\varphi_{p}:[0,1] \rightarrow[0,1]$ given by

$$
\varphi_{p}\left(\sum_{n=1}^{\infty} \frac{x_{n}}{2^{n}}\right)=\sum_{n=1}^{\infty} x_{n} p^{n-\sum_{i=1}^{n-1} x_{i}}(1-p)^{\sum_{i=1}^{n-1} x_{i}},
$$

where $x_{n} \in\{0,1\}$ for every $n \in \mathbb{N}$, is an increasing and continuous solution of (e) satisfying (1). Moreover, $\varphi_{p}$ is singular for every $p \neq \frac{1}{2}$.

The proof of Theorem 1.1, and proofs of its generalizations obtained in $[16,17]$ for a much more general functional equation than (e), are based deeply on self-similar measures.

Throughout this paper, for every $p \in(0,1)$ the symbol $\varphi_{p}$ will be reserved for the function $\varphi_{p}:[0,1] \rightarrow \mathbb{R}$ given by $(2)$. Put

$$
\mathcal{W}_{0}=\operatorname{conv}\left(\left\{\varphi_{p} \mid p \in(0,1)\right\}\right),
$$

where $\operatorname{conv}(A)$ denotes the convex hull of a set $A$, and

$$
\begin{gathered}
\mathcal{C}=\{\varphi:[0,1] \rightarrow[0,1] \mid \varphi \text { is a continuous and increasing solution } \\
\text { of (e) satisfying (1) }\} .
\end{gathered}
$$

From Theorem 1.1 we have $\mathcal{W}_{0} \subset \mathcal{C}$ and, so far, no element of the set $\mathcal{C}$ outside of the set $\mathcal{W}_{0}$ was known.

The main purpose of this paper is to show that

$$
\mathcal{C} \backslash \mathcal{W}_{0} \neq \emptyset
$$

To do this, we need a new approach for studying the set $\mathcal{C}$. The new approach is rooted in a paper by de Rham, published long before either the above problems was posed.

\section{Application of a result by de Rham}

We begin this section by answering both problems based on the following well known result of de Rham.

Theorem 2.1. (see [4]; cf. [9]) For every $p \in(0,1)$ there exists exactly one bounded function $R_{p}:[0,1] \rightarrow \mathbb{R}$ such that

$$
\begin{cases}R_{p}\left(\frac{x}{2}\right)=p R_{p}(x) & \text { for every } x \in[0,1], \\ R_{p}\left(\frac{x+1}{2}\right)=(1-p) R_{p}(x)+p & \text { for every } x \in[0,1]\end{cases}
$$

Moreover: 
(i) $R_{p}$ is continuous, strictly increasing and singular for every $p \neq \frac{1}{2}$.

(ii) $R_{\frac{1}{2}}=\operatorname{id}_{[0,1]}$.

(iii) $R_{p}(0)=0$ and

$$
R_{p}\left(\sum_{n=0}^{\infty} 2^{-\gamma_{n}}\right)=\sum_{n=0}^{\infty}\left(\frac{1-p}{p}\right)^{n} p^{\gamma_{n}}
$$

for every strictly increasing sequence $\left(\gamma_{n}\right)_{n \in \mathbb{N}_{0}}$ of positive integer numbers.

From now on, for every $p \in(0,1)$ the symbol $R_{p}$ will be reserved for the function $R_{p}:[0,1] \rightarrow \mathbb{R}$ given by (4).

Fix $p \in(0,1)$. Adding the equations occurring in $(3)$, we get

$$
R_{p}\left(\frac{x}{2}\right)+R_{p}\left(\frac{x+1}{2}\right)=R_{p}(x)+p
$$

for every $x \in[0,1]$. Putting $x=1$ in both equations of $(3)$, we obtain from the second one $R_{p}(1)=1$ and then from the first one $R_{p}\left(\frac{1}{2}\right)=p$. Moreover, the representation (2) of $\varphi_{p}$ coincides with the representation (4) of $R_{p}$ (see [13, page 268]; see also [11, formula (6)]), and hence

$$
R_{p}=\varphi_{p}
$$

for every $p \in(0,1)$. In consequence,

$$
\mathcal{W}_{0}=\operatorname{conv}\left(\left\{R_{p} \mid p \in(0,1)\right\}\right) .
$$

Let us mention that the functions $R_{p}$ were investigated at the beginning of the 20th century in $[3,6,18]$; for more details see [9].

According to (5) we do not have a new solution to the Matkowski and Wesołowski problem yet. To find $\varphi \in \mathcal{C} \backslash \mathcal{W}_{0}$ we need two results. The first one is due to Hata and Yamaguti and the second one is due to Berg and Krüppel. Before we formulate these results, we define the function $\Phi:(0,1) \times[0,1] \rightarrow$ $[0,1]$ by putting

$$
\Phi(p, x)=R_{p}(x) .
$$

From (5) we see that for all $p \in(0,1)$ and $x \in[0,1]$ we also have

$$
\Phi(p, x)=\varphi_{p}(x) .
$$

We will use both of the above formulas for $\Phi$, depending on the needs in the given situation.

Theorem 2.2. (see [8, page 195]; see also [12, Proposition 3.1]) The function $\Phi$ is differentiable with respect to the first variable. 
Theorem 2.3. (see [2, Theorem 2.1]) The function $\Phi$ is Hölder continuous with respect to the second variable. More precisely, there is $C>0$ such that for every $p \in(0,1)$ we have

$$
|\Phi(p, x)-\Phi(p, y)| \leq C|x-y|^{\alpha_{p}}
$$

for all $x, y \in[0,1]$, where

$$
\alpha_{p}=\min \left\{-\frac{\log p}{\log 2},-\frac{\log (1-p)}{\log 2}\right\} .
$$

For every $a \in(0,1]$ we define the function $\phi_{a}:[0,1] \rightarrow[0,1]$ by putting

$$
\phi_{a}(x)=\frac{1}{a} \int_{0}^{a} \Phi(p, x) d p .
$$

The symbol $\phi_{a}$ will be reserved for the just defined function. Note that (2) gives

$$
\begin{aligned}
\phi_{a}\left(\frac{1}{2^{m}}\right) & =\frac{1}{a} \int_{0}^{a} \varphi_{p}\left(\frac{1}{2^{m}}\right) d p=\frac{1}{a} \int_{0}^{a} p^{m} d p=\frac{a^{m}}{m+1}, \\
\phi_{a}\left(1-\frac{1}{2^{m}}\right) & =\frac{1}{a} \int_{0}^{a}\left(1-(1-p)^{m}\right) d p=1-\frac{1-(1-a)^{m+1}}{(m+1) a}
\end{aligned}
$$

for all $a \in(0,1]$ and $m \in \mathbb{N}_{0}$.

We are now in the position to prove the main result of this paper, which reads as follows.

Theorem 2.4. Assume $a \in(0,1]$. Then $\phi_{a} \in \mathcal{C} \backslash \operatorname{conv}\left(\mathcal{W}_{0} \cup\left\{\phi_{b} \mid b \in(0,1) \backslash\right.\right.$ $\{a\}\})$. Moreover, $\phi_{a}$ is not Hölder continuous.

Proof. From Theorem 1.1 we see that $\phi_{a}$ is an increasing function satisfying (e) for every $x \in[0,1]$ with $\phi_{a}(0)=0$ and $\phi_{a}(1)=1$.

Fix $x, y \in[0,1]$. By Theorem 2.3 we have

$$
\left|\phi_{a}(x)-\phi_{a}(y)\right| \leq \frac{1}{a} \int_{0}^{a}|\Phi(p, x)-\Phi(p, y)| d p \leq \frac{C}{a} \int_{0}^{a}|x-y|^{\alpha_{p}} d p .
$$

If $a \in\left(\frac{1}{2}, 1\right]$, then

$$
\begin{aligned}
\int_{0}^{a}|x-y|^{\alpha_{p}} d p & =\int_{0}^{\frac{1}{2}}|x-y|^{-\frac{\log (1-p)}{\log 2}} d p+\int_{\frac{1}{2}}^{a}|x-y|^{-\frac{\log p}{\log 2}} d p \\
& =\int_{0}^{\frac{1}{2}}(1-p)^{-\frac{\log |x-y|}{\log 2}} d p+\int_{\frac{1}{2}}^{a} p^{-\frac{\log |x-y|}{\log 2}} d p \\
& =\frac{1}{1-\frac{\log |x-y|}{\log 2}}\left[1+a^{1-\frac{\log |x-y|}{\log 2}}-2^{\left.\frac{\log |x-y|}{\log 2}\right] .}\right.
\end{aligned}
$$


If $a \in\left(0, \frac{1}{2}\right]$, then a similar calculation gives

$$
\int_{0}^{a}|x-y|^{\alpha_{p}} d p=\frac{1}{1-\frac{\log |x-y|}{\log 2}}\left[1-a^{1-\frac{\log |x-y|}{\log 2}}\right] .
$$

This implies that $\phi_{a}$ is continuous, and hence that $\phi_{a} \in \mathcal{C}$.

Now we want to prove that $\phi_{a} \notin \operatorname{conv}\left(\mathcal{W}_{0} \cup\left\{\phi_{b} \mid b \in(0,1) \backslash\{a\}\right\}\right)$. Suppose, towards a contradiction, that this is false. Then there are numbers $N \in \mathbb{N}$, $\alpha_{1}, \ldots, \alpha_{N}, \beta_{1}, \ldots, \beta_{N} \in[0,1], p_{1}, \ldots, p_{N} \in(0,1)$ and $b_{1}, \ldots, b_{N} \in(0,1) \backslash\{a\}$ such that $\sum_{n=1}^{N}\left(\alpha_{n}+\beta_{n}\right)=1$ and

$$
\phi_{a}=\sum_{n=1}^{N}\left(\alpha_{n} \varphi_{p_{n}}+\beta_{n} \phi_{b_{n}}\right)
$$

Evaluating the above equation at $x=\frac{1}{2^{m}}$ yields, jointly with (7) and (2),

$$
\frac{a^{m}}{m+1}=\sum_{n=1}^{N}\left(\alpha_{n}\left(p_{n}\right)^{m}+\beta_{n} \frac{\left(b_{n}\right)^{m}}{m+1}\right)
$$

for every $m \in \mathbb{N}$. Dividing both sides of the above equality by $\frac{a^{m}}{m+1}$, and then passing with $m$ to $\infty$ we obtain

$$
1=\sum_{n=1}^{N}\left(\alpha_{n} \lim _{m \rightarrow \infty}(m+1)\left(\frac{p_{n}}{a}\right)^{m}+\beta_{n} \lim _{m \rightarrow \infty}\left(\frac{b_{n}}{a}\right)^{m}\right) \in\{0, \infty\},
$$

a contradiction.

It remains to prove that $\phi_{a}$ is not Hölder continuous. Suppose towards a contradiction that there are $\alpha, C>0$ such that $\left|\phi_{a}(x)-\phi_{a}(y)\right| \leq C|x-y|^{\alpha}$ for all $x, y \in[0,1]$. Then making use of $(8)$, we get

$$
\frac{C}{2^{\alpha m}} \geq\left|\phi_{a}(1)-\phi_{a}\left(1-\frac{1}{2^{m}}\right)\right|=\frac{1-(1-a)^{m+1}}{(m+1) a}
$$

for every $m \in \mathbb{N}$, a contradiction.

\section{Singularity of solutions}

The following lemma is a crucial point in this section.

Lemma 3.1. Assume that $\varphi \in \mathcal{C}$. If $\liminf _{x \rightarrow 0+} \frac{\varphi(x)}{x}=0$, then $\varphi$ is singular.

Proof. According to [16, Remark 2.2] both the absolutely continuous and the singular parts of $\varphi$ satisfy (e) for every $x \in[0,1]$. Comparing this with $[16$, Remark 2.1] we see that the class $\mathcal{C}$ is determined by two of its subclasses $\mathcal{C}_{a}$ and $\mathcal{C}_{s}$, consisting of all absolutely continuous and all singular functions, respectively. From assertion $(i)$ of Theorem 1.1 we know that the class $\mathcal{C}_{a}$ 
consist of exactly one function, and it is the identity on $[0,1]$. Hence there are $\alpha \in[0,1]$ and $\varphi_{\text {sing }} \in \mathcal{C}_{s}$ such that

$$
\varphi(x)=\alpha x+(1-\alpha) \varphi_{\text {sing }}(x)
$$

for every $x \in[0,1]$. Therefore, $\varphi^{\prime}(x)=\alpha$ for almost all $x \in[0,1]$. Since $\varphi_{\text {sing }}([0,1]) \subset[0,1]$, we have

$$
0=\liminf _{x \rightarrow 0+} \frac{\varphi(x)}{x}=\alpha+(1-\alpha) \liminf _{x \rightarrow 0+} \frac{\varphi_{\text {sing }}(x)}{x} \geq \alpha,
$$

which completes the proof.

Now, we are in a position to prove that for every $a \in(0,1]$ the function $\phi_{a}$ is singular. Next, we will have a closer look at the set where its derivative is zero and the set where its derivative is infinite.

Theorem 3.2. For every $a \in(0,1]$ the function $\phi_{a}$ is strictly increasing and singular.

Proof. The strict monotonicity of $\phi_{a}$ is clear. It remains to prove that $\phi_{a}$ is singular.

Assume first that $a \in\left(0, \frac{1}{2}\right]$. Then, making use of $(7)$, we obtain

$$
\liminf _{h \rightarrow 0+} \frac{\phi_{a}(h)}{h} \leq \limsup _{n \rightarrow \infty} 2^{n} \phi_{a}\left(\frac{1}{2^{n}}\right)=\limsup _{n \rightarrow \infty} \frac{(2 a)^{n}}{n+1}=0 .
$$

Therefore, $\phi_{a}$ is singular, by Lemma 3.1.

Assume now that $a \in\left(\frac{1}{2}, 1\right]$. Since $\phi_{\frac{1}{2}}$ is singular, what we have just proved, it follows that $\phi_{\frac{1}{2}}^{\prime}(x)=\phi_{\frac{1}{2}}^{\prime}(1-x)=0$ for almost all $x \in[0,1]$. Fix $x \in[0,1]$ such that $\phi_{\frac{1}{2}}^{\prime}(x)=\phi_{\frac{1}{2}}^{\prime}(1-x)=0$. From [2, Proposition 2.3] we know that

$$
\varphi_{p}(y)=1-\varphi_{1-p}(1-y)
$$

for all $p \in(0,1)$ and $y \in[0,1]$. This jointly with the monotonicity of $\phi_{a}$ and the change of variable theorem gives

$$
\begin{aligned}
0 & \leq \frac{\phi_{a}(x+h)-\phi_{a}(x)}{h}=\frac{1}{a} \int_{0}^{a} \frac{\varphi_{p}(x+h)-\varphi_{p}(x)}{h} d p \\
& =\frac{1}{2 a} \frac{\phi_{\frac{1}{2}}(x+h)-\phi_{\frac{1}{2}}(x)}{h}+\frac{1}{a} \int_{\frac{1}{2}}^{a} \frac{\varphi_{p}(x+h)-\varphi_{p}(x)}{h} d p \\
& =\frac{1}{2 a} \frac{\phi_{\frac{1}{2}}(x+h)-\phi_{\frac{1}{2}}(x)}{h}+\frac{1}{a} \int_{\frac{1}{2}}^{a} \frac{\varphi_{1-p}(1-x)-\varphi_{1-p}(1-x-h)}{h} d p \\
& =\frac{1}{2 a} \frac{\phi_{\frac{1}{2}}(x+h)-\phi_{\frac{1}{2}}(x)}{h}+\frac{1}{a} \int_{1-a}^{\frac{1}{2}} \frac{\varphi_{p}(1-x)-\varphi_{p}(1-x-h)}{h} d p
\end{aligned}
$$




$$
\begin{aligned}
& \leq \frac{1}{2 a} \frac{\phi_{\frac{1}{2}}(x+h)-\phi_{\frac{1}{2}}(x)}{h}+\frac{1}{a} \int_{0}^{\frac{1}{2}} \frac{\varphi_{p}(1-x)-\varphi_{p}(1-x-h)}{h} d p \\
& =\frac{1}{2 a}\left(\frac{\phi_{\frac{1}{2}}(x+h)-\phi_{\frac{1}{2}}(x)}{h}+\frac{\phi_{\frac{1}{2}}(1-x)-\phi_{\frac{1}{2}}(1-x-h)}{h}\right)
\end{aligned}
$$

for every $h \in(0,1]$ with $x+h \in[0,1]$. Passing now with $h$ to 0 we obtain $\phi_{a}^{\prime}(x)=0$.

Before we formulate the next result, let us introduce some notions.

Assume that $x=\sum_{k=1}^{\infty} \frac{x_{k}}{2^{k}}$, where $x_{k} \in\{0,1\}$ for every $k \in \mathbb{N}$. If $x$ has two representations, we take the one with only finitely many $x_{k}$ non-vanishing. For all $n \in \mathbb{N}$ and $p, a \in(0,1)$ we put

$$
I_{n}=\sum_{k=1}^{n-1} x_{k}, \quad D_{1}(x)=\lim _{n \rightarrow \infty} \frac{I_{n}}{n}, \quad D_{0}(x)=1-D_{1}(x) .
$$

Moreover, for any increasing function $f:[0,1] \rightarrow[0,1]$ we put

$$
f_{+}^{\prime}(x)=\lim _{h \rightarrow 0+} \frac{f(x+h)-f(x)}{h} \text { and } f_{-}^{\prime}(x)=\lim _{h \rightarrow 0-} \frac{f(x+h)-f(x)}{h},
$$

assuming that the limits make sense.

Our analysis of the sets where the derivative is 0 or $\infty$ is based on the idea of the proof of the following result, which is due to Kawamura.

Theorem 3.3. (see [11, Theorem 2.2]) Assume that $x \in[0,1]$.

(i) If $x$ is dyadic and $p \in\left(0, \frac{1}{2}\right)$, then $R_{p+}^{\prime}(x)=0$ and $R_{p-}^{\prime}(x)=\infty$.

(ii) If $x$ is dyadic and $p \in\left(\frac{1}{2}, 1\right)$, then $R_{p-}^{\prime}(x)=0$ and $R_{p+}^{\prime}(x)=\infty$.

(iii) If $x$ is not dyadic and $0<D_{1}(x)<1$, then

$$
R_{p}^{\prime}(x)= \begin{cases}0, & \text { if } p^{D_{0}(x)}(1-p)^{D_{1}(x)}<\frac{1}{2}, \\ \infty, & \text { if } p^{D_{0}(x)}(1-p)^{D_{1}(x)}>\frac{1}{2}\end{cases}
$$

Now we can prove the following announced result.

Theorem 3.4. Assume that $x \in[0,1]$.

(i) If $x$ is dyadic and $a \in\left(0, \frac{1}{2}\right)$, then $\phi_{a+}^{\prime}(x)=0$ and $\phi_{a-}^{\prime}(x)=\infty$.

(ii) If $x$ is dyadic and $a \in\left(\frac{1}{2}, 1\right)$, then $\phi_{a}^{\prime}(x)=\infty$.

(iii) If $x$ is not dyadic with $D_{0}(x) \in(0,1)$ and $a \in\left(0, D_{0}(x)\right)$, then

$$
\phi_{a}^{\prime}(x)= \begin{cases}0, & \text { if } p^{D_{0}(x)}(1-p)^{D_{1}(x)}<\frac{1}{2} \text { for all } p \leq a, \\ \infty, & \text { if } p^{D_{0}(x)}(1-p)^{D_{1}(x)}>\frac{1}{2} \text { for some } p \leq a .\end{cases}
$$

Proof. We follow the idea of the proof of [11, Theorem 2.2] by Kawamura; note that our definition of $I_{n}$ differs from that of [11], because we only sum up to $n-1$ instead of $n$ as Kawamura does.

Fix $a \in(0,1)$ and $x=\sum_{k=1}^{\infty} \frac{x_{k}}{2^{k}}$, where $x_{k} \in\{0,1\}$ for every $k \in \mathbb{N}$. 
Let us first consider all the cases where $\phi_{a-}^{\prime}(x)$ or $\phi_{a+}^{\prime}(x)$ vanishes.

We begin with the cases where $\phi_{a+}^{\prime}(x)=0$. Observe that in each of these cases Theorem 3.3 yields $R_{p+}^{\prime}(x)=0$ for almost all $p \in(0, a)$. So if we could apply the Lebesgue dominated convergence theorem, we would get

$$
0 \leq \phi_{a+}^{\prime}(x)=\lim _{h \rightarrow 0+} \frac{1}{a} \int_{0}^{a} \frac{\Phi(p, x+h)-\Phi(p, x)}{h} d p=\frac{1}{a} \int_{0}^{a} R_{p+}^{\prime}(x) d p=0 .
$$

Therefore, we see that to conclude the proof, it is enough to verify that

$$
(p, h) \mapsto \frac{\varphi_{p}(x+h)-\varphi_{p}(x)}{h}
$$

is bounded almost everywhere from above on $(0,1) \times(0,1-x)$ by a constant.

Let us start the required verification with the dyadic case of $x$, i.e. where there exists $N \in \mathbb{N}$ such that $x_{k}=0$ for every $k>N$. Then

Fix $k \in \mathbb{N}$ and $h \in \mathbb{R}$ such that $k>N, \frac{k-I_{N+1}}{k}>a$ and $2^{-(k+1)} \leq h<2^{-k}$.

$\frac{\varphi_{p}(x+h)-\varphi_{p}(x)}{h} \leq 2^{k+1}\left(\varphi_{p}\left(x+2^{-k}\right)-\varphi_{p}(x)\right)=2^{k+1} p^{k-I_{N+1}}(1-p)^{I_{N+1}}$.

As the polynomial $p^{k-I_{N+1}}(1-p)^{I_{N+1}}$ is increasing on the interval $\left[0, \frac{k-I_{N+1}}{k}\right]$ and $p \leq a$, we have

$$
\frac{\varphi_{p}(x+h)-\varphi_{p}(x)}{h} \leq 2^{k+1} a^{k-I_{N+1}}(1-a)^{I_{N+1}} .
$$

Since $\lim _{k \rightarrow \infty} 2^{k+1} a^{k-I_{N+1}}(1-a)^{I_{N+1}}=\lim _{k \rightarrow \infty} 2 \cdot(2 a)^{k}\left(\frac{1-a}{a}\right)^{I_{N+1}}=0$, we see that the sequence $\left(2^{k+1} a^{k-I_{N+1}}(1-a)^{I_{N+1}}\right)_{k \in \mathbb{N}}$ is bounded. In consequence

$$
\frac{\Phi(p, x+h)-\Phi(p, x)}{h} \leq \sup \left\{2^{k+1} a^{k-I_{N+1}}(1-a)^{I_{N+1}} \mid k \in \mathbb{N}\right\}<\infty .
$$

Thus we have proved the first part of assertion $(i)$.

Now, we pass to the case where $x$ is not dyadic and $p^{D_{0}(x)}(1-p)^{D_{1}(x)}<\frac{1}{2}$ for every $p \leq a$.

Let $\left(n_{k}\right)_{k \in \mathbb{N}}$ be the subsequence of all natural numbers for which $x_{n_{k}}=0$; i.e. $n_{k}$ is the address of the $k$-th 0 in the binary expansion of $x$. Clearly,

$$
\lim _{k \rightarrow \infty} \frac{n_{k+1}}{n_{k}}=1 \text {. }
$$

For every $k \in \mathbb{N}$ we have $I_{n_{k}}=n_{k}-k$, and further for every $m \in \mathbb{Z}$ we also have

$$
\lim _{k \rightarrow \infty} \frac{k-m}{n_{k}}=D_{0}(x) .
$$

Fix $p \in(0,1)$ (we will later restrict ourselves to $p \leq a$ ) and $k \in \mathbb{N}$, and let $h$ be such that $2^{-n_{k+1}} \leq h<2^{-n_{k}}$. Then 


$$
\begin{aligned}
\frac{\varphi_{p}(x+h)-\varphi_{p}(x)}{h} \leq & 2^{n_{k+1}}\left(\varphi_{p}\left(x+2^{-n_{k}}\right)-\varphi_{p}(x)\right) \\
= & 2^{n_{k+1}}\left[p^{k}(1-p)^{n_{k}-k}\right. \\
& \left.+\frac{1-2 p}{p} \sum_{n=n_{k}+1}^{\infty} x_{n} p^{n-I_{n}}(1-p)^{I_{n}}\right] .
\end{aligned}
$$

Let $(n(l))_{l \in \mathbb{N}}$ be the subsequence of all natural numbers greater than $n_{k}$ for which $x_{n(l)}=1$; i.e. $n(l)$ is the address of the $l$-th 1 appearing after position $n_{k}$ in the binary expansion of $x$. As $n(l)-n_{k}-l \geq 0$ for every $l \in \mathbb{N}$, we have

$$
\begin{aligned}
\sum_{n=n_{k}+1}^{\infty} x_{n} p^{n-I_{n}}(1-p)^{I_{n}} & =p^{k}(1-p)^{n_{k}-k} \sum_{l=1}^{\infty} p^{n(l)-n_{k}-l+1}(1-p)^{l-1} \\
& \leq p^{k+1}(1-p)^{n_{k}-k} \sum_{l=1}^{\infty}(1-p)^{l-1}=p^{k}(1-p)^{n_{k}-k}
\end{aligned}
$$

Thus

$$
\begin{aligned}
\frac{\varphi_{p}(x+h)-\varphi_{p}(x)}{h} & \leq 2^{n_{k+1}} p^{k}(1-p)^{n_{k}-k}\left(1+\frac{1-2 p}{p}\right) \\
& =2^{n_{k+1}} p^{k}(1-p)^{n_{k}-k}\left(\frac{1-p}{p}\right) \\
& =2^{n_{k+1}} p^{k-1}(1-p)^{n_{k}-k+1} .
\end{aligned}
$$

As $a<D_{0}(x)$, we conclude from (12) that there is $k_{0} \in \mathbb{N}$ such that $a \leq \frac{k-1}{n_{k}}$ for every $k \in \mathbb{N}$ with $k \geq k_{0}$. Assume now that $k \geq k_{0}$ and that $p \leq a$. As the polynomial $p^{k-1}(1-p)^{n_{k}-k+1}$ is increasing on the interval $\left[0, \frac{k-1}{n_{k}}\right]$ and $p \leq a$, we have

$$
\frac{\varphi_{p}(x+h)-\varphi_{p}(x)}{h} \leq 2^{n_{k+1}} a^{k-1}(1-a)^{n_{k}-k+1} .
$$

Applying (11) and (12), we obtain

$$
\lim _{k \rightarrow \infty} 2^{\frac{n_{k+1}}{n_{k}}} a^{\frac{k-1}{n_{k}}}(1-a)^{1-\frac{k-1}{n_{k}}}=2 a^{D_{0}(x)}(1-a)^{D_{1}(x)}<1,
$$

and hence

$$
\lim _{k \rightarrow \infty} 2^{n_{k+1}} a^{k-1}(1-a)^{n_{k}-k+1}=\lim _{k \rightarrow \infty}\left(2^{\frac{n_{k+1}}{n_{k}}} a^{\frac{k-1}{n_{k}}}(1-a)^{1-\frac{k-1}{n_{k}}}\right)^{n_{k}}=0 .
$$

This implies that the sequence $\left(2^{n_{k+1}} a^{k-1}(1-a)^{n_{k}-k+1}\right)_{k \in \mathbb{N}}$ is bounded. In consequence

$$
\frac{\Phi(p, x+h)-\Phi(p, x)}{h} \leq \sup \left\{2^{n_{k+1}} a^{k-1}(1-a)^{n_{k}-k+1} \mid k \in \mathbb{N}\right\}<\infty .
$$


Thus we have proved that $\phi_{a+}^{\prime}(x)=0$ in the case where $x$ is not dyadic and $p^{D_{0}(x)}(1-p)^{D_{1}(x)}<\frac{1}{2}$ for every $p \leq a$, which is the first step of the proof of assertion (iii).

We stay in the same case of assertion (iii), i.e. we assume that $x$ is not dyadic and $p^{D_{0}(x)}(1-p)^{D_{1}(x)}<\frac{1}{2}$ for every $p \leq a$. But now we want to prove that $\phi_{a-}^{\prime}(x)=0$.

Observe first that (9) yields

$$
\begin{aligned}
\phi_{a-}^{\prime}(x) & =\lim _{h \rightarrow 0+} \frac{1}{a} \int_{0}^{a} \frac{\varphi_{p}(x)-\varphi_{p}(x-h)}{h} d p \\
& =\frac{1}{a} \lim _{h \rightarrow 0+} \int_{0}^{a} \frac{\varphi_{1-p}(1-x+h)-\varphi_{1-p}(1-x)}{h} d p \\
& =\frac{1}{a} \lim _{h \rightarrow 0+} \int_{1-a}^{1} \frac{\varphi_{p}(1-x+h)-\varphi_{p}(1-x)}{h} d p \\
& =\frac{1}{a} \lim _{h \rightarrow 0+} \int_{1-a}^{1} \frac{\Phi(p, 1-x+h)-\Phi(p, 1-x)}{h} d p .
\end{aligned}
$$

Since in the considered case Theorem 3.3 implies that $R_{p+}^{\prime}(1-x)=0$ for almost all $p \in(1-a, 1)$ as we will verify (in a moment) in (16), the idea is to follow the proof of $\phi_{a+}(x)=0$, replacing $x$ by $1-x$ with suitable changes, which we explain in details.

Fix $1-a<p<1$. Since $D_{0}(1-x)=D_{1}(x)$, we have

$$
p^{D_{0}(1-x)}(1-p)^{D_{1}(1-x)}=p^{D_{1}(x)}(1-p)^{D_{0}(x)}<\frac{1}{2} .
$$

In the following $n_{k}$ refers to the address of the $k$-th 0 in the binary expansion of $1-x$ instead of $x$. Using (12) for $1-x$, instead of $x$, we see that there exists $k_{0} \in \mathbb{N}$ such that $1-a \geq \frac{k}{n_{k}}$ for every $k \in \mathbb{N}$ with $k \geq k_{0}$. Fix $k \geq k_{0}$ and let $h$ be such that $2^{-n_{k+1}} \leq h<2^{-n_{k}}$. We have shown before (see (13)) that

$$
\frac{\varphi_{p}(1-x+h)-\varphi_{p}(1-x)}{h} \leq 2^{n_{k+1}} p^{k-1}(1-p)^{n_{k}+1-k} .
$$

As the polynomial $p^{k-1}(1-p)^{n_{k}+1-k}$ is decreasing on the interval $\left[\frac{k}{n_{k}}, 1\right]$ and $p \geq 1-a$, inequality (14) holds with $a$ replaced by $1-a$, and hence also (15) holds with $a$ replaced by $1-a$, which is what we need to apply (10) and conclude that $\phi_{a-}^{\prime}(x)=0$.

It remains to prove all the cases where a one-sided derivative of $\phi_{a}$ at the point $x$ equals $\infty$. We prove these cases in more or less one go.

First of all, we show that in each of these cases for every $N \in \mathbb{N}$ we can find an open interval $L_{N}$ having 0 as its right or left endpoint (depending on the one-sided derivative of $\phi_{a}$ at the point $x$ we are interested in) and a Lebesgue 
measurable set $P_{N} \subset(0, a)$ such that

$$
\frac{\varphi_{p}(x+h)-\varphi_{p}(x)}{h} \geq N \quad \text { for all } p \in P_{N} \text { and } h \in L_{N}
$$

and

$$
\lim _{N \rightarrow \infty} N\left|P_{N}\right|=\infty
$$

where $\left|P_{N}\right|$ denotes the Lebesgue measure of the set $P_{N}$.

In the case $(i)$, we apply assertion $(i)$ of Theorem 3.3 to find $L_{N}$ having 0 as right endpoint and $P_{N} \subset\left(0, \frac{1}{2}\right)$; the same is true in the case $(i i)$, and moreover, by assertion ( $i$ i) of Theorem 3.3, we can also find $L_{N}$ whose left endpoint is 0 and $P_{N} \subset\left(\frac{1}{2}, 1\right)$. In the case $($ iii) we use assertion ( $i i i)$ of Theorem 3.3 to find $L_{N}$ having 0 as right endpoint as well as to find $L_{N}$ having 0 as left endpoint and $P_{N}$ being the intersection of the interval $(0, a)$ with a neighbourhood of this parameter $p \leq a$ for which $p^{D_{0}(x)}(1-p)^{D_{1}(x)}>\frac{1}{2}$.

If both sets $L_{N}$ and $P_{N}$ satisfy the above conditions, then

$$
\frac{\phi_{a}(x+h)-\phi_{a}(x)}{h} \geq \frac{1}{a} \int_{P_{N}} \frac{\varphi_{p}(x+h)-\varphi_{p}(x)}{h} d p \geq \frac{1}{a} N\left|P_{N}\right|
$$

for all $h \in L_{N}$ and $N \in \mathbb{N}$.

Finally, in each of the considered cases we conclude that the one-sided derivative of $\phi_{a}$ at the point $x$ equals $\infty$.

\section{Continuity of the function $\Phi$}

Before we construct more new solutions to the Matkowski and Wesołowski problem, let us look at the function $\Phi$. By Theorem 2.1 this function is continuous with respect to the second variable, and from Theorem 2.2 we see that it is also continuous with respect to the first variable. Thus the question reads: Is it continuous? To answer this question we need a result due to Grushka.

Theorem 4.1. (see [7]) Let $N \in \mathbb{N}$ and let $a_{n}, b_{n} \in \mathbb{R}$ with $a_{n}<b_{n}$ for every $n \in$ $\{1, \ldots, N+1\}$. If $f:\left(a_{1}, b_{1}\right) \times \cdots \times\left(a_{N}, b_{N}\right) \times\left(a_{N+1}, b_{N+1}\right) \rightarrow \mathbb{R}$ is continuous with respect to each variable separately and monotonous with respect to each of the first $N$ variables separately, then $f$ is continuous.

Theorem 4.2. The function $\Phi$ is continuous.

Proof. According to Theorems 2.1, 2.2 and 4.1 it suffices to prove that $\Phi$ is continuous at every point of the set $(0,1) \times\{0,1\}$.

Fix $p_{0} \in(0,1)$ and $\varepsilon>0$. Next choose $n \in \mathbb{N}$ such that $0<\left(p_{0}+\frac{1}{2^{n}}\right)^{n} \leq \varepsilon$. Fix $(p, x) \in(0,1) \times[0,1]$ and assume that $\left(p-p_{0}\right)^{2}+x^{2} \leq \frac{1}{4^{n}}$. Hence $p \leq p_{0}+\frac{1}{2^{n}}$ 
and $x \leq \frac{1}{2^{n}}$. Then, making use of Theorem 2.1, we obtain

$$
\left|\Phi(p, x)-\Phi\left(p_{0}, 0\right)\right|=R_{p}(x) \leq R_{p}\left(\frac{1}{2^{n}}\right)=p^{n} \leq\left(p_{0}+\frac{1}{2^{n}}\right)^{n} \leq \varepsilon,
$$

which proves that $\Phi$ is continuous at the point $\left(p_{0}, 0\right)$.

Noting that $R_{p}(x)=1-R_{1-p}(1-x)$ for every $x \in[0,1]$, as stated for example in [1, Proposition 2.3], we can easily see that $\Phi$ is continuous at every point of the set $(0,1) \times\{1\}$.

Observe that the function $\Phi$ cannot be extended continuously to the closed unit square $[0,1]^{2}$; indeed

$$
\lim _{p \rightarrow 0} \Phi(p, x)=\left\{\begin{array}{l}
0, \text { if } x \in[0,1) \\
1, \text { if } x=1
\end{array} \quad \text { and } \quad \lim _{p \rightarrow 1} \Phi(p, x)=\left\{\begin{array}{l}
0, \text { if } x=0 \\
1, \text { if } x \in(0,1]
\end{array},\right.\right.
$$

and the continuity breaks at the points $(0,1)$ and $(1,0)$.

\section{More new solutions}

We want to construct more new solutions to the Matkowski and Wesołowski problem different from those presented in Theorem 2.4. For this purpose we fix a Borel probability measure $\mu$ on $(0,1)$. Next, given a Borel measurable function $g:(0,1) \rightarrow(0,1)$, we define a function $\psi_{g}:[0,1] \rightarrow[0,1]$ by putting

$$
\psi_{g}(x)=\int_{(0,1)} \Phi(g(p), x) d \mu(p) ;
$$

note that the function is well defined, because by Theorem 2.2 the map $p \mapsto$ $\Phi(g(p), x)$ is Borel measurable and bounded for every $x \in[0,1]$. We keep the symbol $\psi_{g}$ for the above defined function to the end of this paper.

Given a Borel measurable function $g:(0,1) \rightarrow(0,1)$, we define the nonincreasing rearrangement $g^{*}:(0,1) \rightarrow[0,1]$ of $g$ by putting

$$
g^{*}(t)=\inf \{\lambda \geq 0 \mid \mu(\{p \in(0,1) \mid g(p)>\lambda\}) \leq t\} .
$$

It is clear that the function $g^{*}$ is non-increasing and right-side continuous. From [1, Proposition 2.3] and Theorem 2.2 we deduce that the function $\Phi$ is strictly increasing and continuous with respect to the first variable. Therefore, according to [5, Corollary 3.2.8] we have

$$
\psi_{g}(x)=\int_{(0,1)} \Phi(g(p), x) d \mu(p)=\int_{(0,1)} \Phi\left(g^{*}(p), x\right) d p
$$

for every $x \in[0,1]$.

In view of the above observation let us assume, to the end of this paper, that $\mu$ is the one-dimensional Lebesgue measure. 
Theorem 5.1. Assume that $g:(0,1) \rightarrow(0,1)$ is a Borel measurable function. Then $\psi_{g} \in \mathcal{C}$, and it is strictly increasing.

Proof. From Theorem 1.1 we see that $\psi_{g}$ is a strictly increasing function satisfying (e) for every $x \in[0,1]$ with $\psi_{g}(0)=0$ and $\psi_{g}(1)=1$. To prove that $\psi_{g}$ is continuous we fix $x \in[0,1]$ and a sequence $\left(x_{n}\right)_{n \in \mathbb{N}}$ of elements of $[0,1]$ converging to $x$. Applying the Lebesgue dominated convergence theorem jointly with Theorem 4.2 we obtain, noting that the integrand is bounded,

$$
\lim _{n \rightarrow \infty} \psi_{g}\left(x_{n}\right)=\int_{(0,1)} \lim _{n \rightarrow \infty} \Phi\left(g(p), x_{n}\right) d p=\psi_{g}(x) .
$$

Therefore, $\psi_{g} \in \mathcal{C}$.

Theorem 5.1 produces a very large class of new solutions to the Matkowski and Wesołowski problem.

Put

$$
\mathcal{W}_{1}=\operatorname{conv}\left(\left\{\phi_{a} \mid a \in(0,1)\right\}\right)
$$

and for every $s \in(0, \infty)$ we define the function $g_{s}:(0,1) \rightarrow(0,1)$ by putting

$$
g_{s}(p)=p^{s} ;
$$

the symbol $g_{s}$ will be reserved just for this function. Obviously,

$$
\psi_{g_{s}}\left(\frac{1}{2^{m}}\right)=\frac{1}{s m+1}
$$

for all $s \in(0, \infty)$ and $m \in \mathbb{N}$.

Corollary 5.2. Assume that $\mu$ is the one-dimensional Lebesgue measure. Then for every $s \in(0, \infty)$ we have $\psi_{g_{s}} \in \mathcal{C} \backslash \operatorname{conv}\left(\mathcal{W}_{0} \cup \mathcal{W}_{1} \cup\left\{\psi_{g_{r}} \mid r \in(0, \infty) \backslash\{s\}\right\}\right)$.

Proof. According to Theorem 5.1 we only need to show that $\psi_{g_{s}} \notin \operatorname{conv}\left(\mathcal{W}_{0} \cup\right.$ $\left.\mathcal{W}_{1} \cup\left\{\psi_{g_{r}} \mid r \in(0, \infty) \backslash\{s\}\right\}\right)$ for every $s \in(0, \infty)$.

Fix $s \in(0, \infty)$ and suppose towards a contradiction that there are $N \in \mathbb{N}$, $\alpha_{1}, \ldots, \alpha_{N}, \beta_{1}, \ldots, \beta_{N}, \gamma_{1}, \ldots, \gamma_{N} \in[0,1], p_{1}, \ldots, p_{N}, a_{1}, \ldots, a_{N} \in(0,1)$ and $r_{1}, \ldots, r_{N} \in(0, \infty) \backslash\{s\}$ such that $\sum_{n=1}^{N}\left(\alpha_{n}+\beta_{n}+\gamma_{n}\right)=1$ and

$$
\psi_{g_{s}}=\sum_{n=1}^{N}\left(\alpha_{n} \varphi_{p_{n}}+\beta_{n} \phi_{a_{n}}+\gamma_{n} \psi_{g_{r_{n}}}\right) .
$$

We evaluate the above expression at the point $\frac{1}{2^{m}}$ by applying (17), (2) and (7):

$$
\frac{1}{s m+1}-\sum_{n=1}^{N} \gamma_{n} \frac{1}{r_{n} m+1}=\sum_{n=1}^{N}\left(\alpha_{n}\left(p_{n}\right)^{m}+\beta_{n} \frac{\left(a_{n}\right)^{m}}{m+1}\right)
$$


for every $m \in \mathbb{N}$. Multiplying both sides by $(m+1)(s m+1) \prod_{n=1}^{N}\left(r_{n} m+1\right)$ we find that there are polynomials $P_{1}, P_{2}$ and $P_{3}$ in $m$ such that

$$
P_{1}(m)=\sum_{n=1}^{N}\left(\alpha_{n} P_{2}(m)\left(p_{n}\right)^{m}+\beta_{n} P_{3}(m)\left(a_{n}\right)^{m}\right)
$$

for every $m \in \mathbb{N}$. Let us assume for a contradiction that the leading term of $P_{1}$ is $\alpha_{K} m^{K}$ for some $K \geq 1$. Then dividing both sides by $\alpha_{K} m^{K}$ and letting $m$ tend to $\infty$ we have that $1=0$, which is impossible. Hence $P_{1}$ is constant. Taking the limit as $m$ tends to $\infty$ on the right-hand side of (18) gives $P_{1}=0$. Since $P_{1}(m)=(m+1)\left(\prod_{n=1}^{N}\left(r_{n} m+1\right)+(s m+1) Q(m)\right)$ with a polynomial $Q$ in $m$, it follows that

$$
\prod_{n=1}^{N}\left(r_{n} m+1\right)+(s m+1) Q(m)=0
$$

for every $m \in \mathbb{R}$. Finally, putting $m=-\frac{1}{s}$ in (19) we conclude that $s=r_{n}$ for some $n \in\{1, \ldots, N\}$, a contradiction.

Put

$$
\mathcal{W}_{2}=\operatorname{conv}\left(\left\{\psi_{g_{s}} \mid s \in(0, \infty)\right\}\right)
$$

and for every $\lambda \in(0,1)$ define the function $\sin _{\lambda}:(0,1) \rightarrow(0,1)$ by putting

$$
\sin _{\lambda}(p)=\lambda \sin \pi p .
$$

Again, the symbol $\sin _{\lambda}$ will be reserved for the just defined function. One can check that

$$
\psi_{\sin _{\lambda}}\left(\frac{1}{4^{m}}\right)=\left(\frac{\lambda}{2}\right)^{2 m} \frac{(2 m) !}{(m !)^{2}}
$$

for all $\lambda \in(0,1)$ and $m \in \mathbb{N}$.

Corollary 5.3. Assume that $\mu$ is the one-dimensional Lebesgue measure. Then for every $\lambda \in(0,1)$ we have $\psi_{\sin _{\lambda}} \in \mathcal{C} \backslash \operatorname{conv}\left(\mathcal{W}_{0} \cup \mathcal{W}_{1} \cup \mathcal{W}_{2}\right)$.

Proof. According to Theorem 5.1 we only need to show that $\psi_{\sin _{\lambda}} \notin \operatorname{conv}\left(\mathcal{W}_{0} \cup\right.$ $\left.\mathcal{W}_{1} \cup \mathcal{W}_{2}\right)$

Fix $\lambda \in(0, \infty)$ and suppose towards a contradiction that there are $N \in \mathbb{N}$, $\alpha_{1}, \ldots, \alpha_{N}, \beta_{1}, \ldots, \beta_{N}, \gamma_{1}, \ldots, \gamma_{N} \in[0,1], p_{1}, \ldots, p_{N}, a_{1}, \ldots, a_{N} \in(0,1)$ and $s_{1}, \ldots, s_{N} \in(0, \infty)$ such that $\sum_{n=1}^{N}\left(\alpha_{n}+\beta_{n}+\gamma_{n}\right)=1$ and

$$
\psi_{\sin _{\lambda}}=\sum_{n=1}^{N}\left(\alpha_{n} \varphi_{p_{n}}+\beta_{n} \phi_{a_{n}}+\gamma_{n} \psi_{g_{s_{n}}}\right) .
$$

Applying (20), (2), (7), and (17), we obtain

$$
\left(\frac{\lambda}{2}\right)^{2 m} \frac{(2 m) !}{(m !)^{2}}=\sum_{n=1}^{N}\left(\alpha_{n}\left(p_{n}\right)^{2 m}+\beta_{n} \frac{\left(a_{n}\right)^{2 m}}{2 m+1}+\gamma_{n} \frac{1}{2 s_{n} m+1}\right)
$$


for every $m \in \mathbb{N}$. Dividing both sides of the above equality by $\left(\frac{\lambda}{2}\right)^{2 m} \frac{(2 m) !}{(m !)^{2}}$, and then passing with $m$ to $\infty$ we obtain, by for example using (20),

$$
\begin{aligned}
1= & \lim _{m \rightarrow \infty} \sum_{n=1}^{N}\left(\alpha_{n} \frac{(m !)^{2}}{(2 m) !}\left(\frac{2 p_{n}}{\lambda}\right)^{2 m}+\beta_{n} \frac{(m !)^{2}}{(2 m+1) !}\left(\frac{2 a_{n}}{\lambda}\right)^{2 m}\right. \\
& \left.+\gamma_{n} \frac{(m !)^{2}}{(2 m) !\left(2 s_{n} m+1\right)}\left(\frac{2}{\lambda}\right)^{2 m}\right) \in\{0, \infty\},
\end{aligned}
$$

a contradiction.

\section{Acknowledgements}

The research was supported by the University of Silesia Mathematics Department (Iterative Functional Equations and Real Analysis program).

Open Access. This article is licensed under a Creative Commons Attribution 4.0 International License, which permits use, sharing, adaptation, distribution and reproduction in any medium or format, as long as you give appropriate credit to the original author(s) and the source, provide a link to the Creative Commons licence, and indicate if changes were made. The images or other third party material in this article are included in the article's Creative Commons licence, unless indicated otherwise in a credit line to the material. If material is not included in the article's Creative Commons licence and your intended use is not permitted by statutory regulation or exceeds the permitted use, you will need to obtain permission directly from the copyright holder. To view a copy of this licence, visit http:// creativecommons.org/licenses/by/4.0/.

Publisher's Note Springer Nature remains neutral with regard to jurisdictional claims in published maps and institutional affiliations.

\section{References}

[1] Berg, L., Krüppel, M.: De Rham's singular function and related functions. Z. Anal. Anwendungen 19(1), 227-237 (2000)

[2] Berg, L., Krüppel, M.: De Rham's singular function, two-scale difference equations and Appell polynomials. Results Math. 38(1-2), 18-47 (2000)

[3] Cesáro, E.: Fonctions continues sans dérivée. Archiv der Math. und Phys. 10, 57-63 (1906)

[4] de Rham, G.: Sur quelques courbes definies par des equations fonctionnelles. Univ. e Politec. Torino. Rend. Sem. Mat. 16, 101-113 (1956/1957)

[5] Edmunds, D.E., Evans, W.D.: Hardy Operators, Function Spaces and Embeddings. Springer Monographs in Mathematics. Springer, Berlin (2004)

[6] Faber, G.: Über stetige Funktionen. (Zweite Abhandlung.). Math. Ann. 69, 372-443 (1910)

[7] Grushka, Y.I.: On monotonous separately continuous functions. Appl. Gen. Topol. 20(1), 75-79 (2019)

[8] Hata, M., Yamaguti, M.: The Takagi function and its generalization. Japan J. Appl. Math. 1(1), 183-199 (1984) 
[9] Kairies, H.-H.: Functional equations for peculiar functions. Aequationes Math. 53(3), 207-241 (1997)

[10] Kania, T., Máthé, A., Morawiec, J., Rmoutil, M., Zürcher, T.: A functional equation (2016). Manuscript

[11] Kawamura, K.: On the set of points where Lebesgue's singular function has the derivative zero. Proc. Japan Acad. Ser. A Math. Sci. 87(9), 162-166 (2011)

[12] Krüppel, M.: De Rham's singular function, its partial derivatives with respect to the parameter and binary digital sums. Rostock. Math. Kolloq. 64, 57-74 (2009)

[13] Łomnicki, Z., Ulam, S.: Sur la théorie de la mesure dans les espaces combinatoires et son application au calcul des probabilités. I. Variables indépendantes. Fund. Math. 23(1), $237-278$ (1934)

[14] Matkowski, J.: Remark on BV-solutions of a functional equation connected with invariant measures. Aequationes Math. 29(2-3), 210-213 (1985)

[15] Misiewicz, J., Wesołowski, J.: Winding planar probabilities. Metrika 75(4), 507-519 (2012)

[16] Morawiec, J., Zürcher, T.: On a problem of Janusz Matkowski and Jacek Wesołowski. Aequationes Math. 92(4), 601-615 (2018)

[17] Morawiec, J., Zürcher, T.: On a problem of Janusz Matkowski and Jacek Wesołowski, II. Aequationes Math. 93(1), 91-108 (2019)

[18] Salem, R.: On some singular monotonic functions which are strictly increasing. Trans. Amer. Math. Soc. 53, 427-439 (1943)

Janusz Morawiec and Thomas Zürcher

Instytut Matematyki

Uniwersytet Śląski

Bankowa 14

40-007 Katowice

Poland

e-mail: morawiec@math.us.edu.pl

Thomas Zürcher

e-mail: thomas.zurcher@us.edu.pl

Received: June 8, 2020

Revised: February 3, 2021

Accepted: February 5, 2021 